\title{
DETERMINATION OF CAFFEINE CONTENT IN NON-ALCOHOLIC BEVERAGES AND ENERGY DRINKS USING HPLC-UV METHOD
}

\section{Muriira Geoffrey Karau', James N Kihunyu², Nahashon M Kathenya1, Laura N Wangai ${ }^{3}$, Daniel Kariuki ${ }^{3}$, \& Rotich Henry Kibet ${ }^{1}$}

\author{
${ }^{1}$ Department of Research and Development, \\ Kenya Bureau of Standards, Nairobi, Kenya; \\ ${ }^{2}$ Department of Chemical Science and Technology, \\ Kenya Polytechnic University College, Nairobi, Kenya; \\ ${ }^{3}$ Jomo Kenyatta University of Agriculture and Technology, \\ Department of Biochemistry and Molecular Biology, Nairobi, Kenya
}

\begin{abstract}
The purpose of this study was to determine the amount of caffeine in non-alcoholic energy drinks and prepared teas using reverse phase HPLC. Caffeine was extracted from 19 different types of non-alcoholic beverages and prepared teas sampled from supermarkets in Nairobi Central Business District, Kenya. These were analyzed alongside a caffeine standard of $99 \%$ purity by use of HPLC-UV detector at the wavelength of $272 \mathrm{~nm}$, Supelco HS C18 column $25 \mathrm{~cm}$ x 4.6 $\mathrm{cm} \times 5 \mu \mathrm{m}$, oven temperature of $40 \mathrm{oC}$, mobile phase 80:20 (v/v) of methanol: water and mobile phase flow rate of $1.5 \mathrm{~mL} / \mathrm{min}$. For quantitation purposes, serial dilution of the caffeine standard gave correlation coefficient (r) of 0.9993 and the retention time of $2.11 \pm 0.03$ minute. Percentage recovery of caffeine from the column ranged from 89.78 to $105.59 \%$. Limits of detection and quantitation were found to be 0.279 and $0.931 \mu \mathrm{g} / \mathrm{mL}$ respectively. It was found that Burn ${ }^{\circledR}, \mathrm{XL}$ energy drink ${ }^{\circledR}$ and Red Bull ${ }^{\circledR}$ had the highest amount of caffeine. It was however noted that though most of the non-alcoholic beverages had high caffeine content they had no label claim.
\end{abstract}

Key Words: Reverse Phase High Performance Liquid Chromatography (HPLC), Ultra violet visible (UV/VIS), caffeine, non-alcoholic beverages

\section{INTRODUCTION}

Caffeine, the common name for 1,3 , 7-trimethylxanthine, is the most widely used psychoactive drug in the world (Barone \& Roberts, 1996). Approximately $80 \%$ of the world's population consumes it on a daily basis, and continuous research is being carried out to determine its health benefits and consequences. The amount of caffeine consumed daily in foods and beverages varies widely. A typical cup of brewed coffee contains approximately $100 \mathrm{mg}$ of caffeine and a cup of green tea contains 20$30 \mathrm{mg}$ of caffeine. Caffeine consumption from

Corresponding author: Muriira Geoffrey, P. O Box 54974-00200, Nairobi, Kenya. Tel. No:. +254722508443;

Email:karaugm2@gmail.com 
all sources reaches $210-238 \mathrm{mg} /$ person per day in the USA and Canada and more than 400mg per person per day in Sweden and Finland (Klatsky et al., 1993; Barone \& Roberts, 1996).

Caffeine acts as a mild central nervous system stimulant (Chou \& Bell, 2007), which is completely absorbed after ingestion within 30 to 45 minutes and its effects substantially diminish within about 3 hours. Coffee, tea and soft drinks are the major sources of caffeine in the diets of US adults, with coffee being the primary and most potent source (Knight et al., 2004; Frary et al., 2005). Many over-the-counter medications, such as cold and allergy tablets, headache medicines, diuretics, and stimulants also contain caffeine, although they lead to relatively minimal intake (Schreiber et al., 1988).

Caffeine is a central nervous system and metabolic stimulant (Nehling et al., 1992) and is used both recreationally and medically to reduce physical fatigue and restore mental alertness when unusual weakness or drowsiness occurs. Caffeine stimulates the central nervous system first at the higher levels, resulting in increased alertness and wakefulness, faster and clearer flow of thought, increased focus, and better general body coordination, and later at the spinal cord level at higher doses (Bolton \& Gary, 1981).

Some researchers have however suggested several psychiatric syndromes that are associated with high caffeine consumption, among them anxiety disorders, diuretic effects, caffeine induced sleep disorder, caffeine intoxication, caffeine withdrawal, and caffeine dependency syndrome (Winkelmayer et al., 2005). Caffeine has been associated with coronary heart disease (Yano et al., 1977), cardiovascular diseases (Dawber et al., 1974), hypertension and hypoglycemia (Watson \& Kerr, 1999; Winkelmayer et al., 2005).

There is a growing individual desire to know the amount of caffeine consumed from non-alcoholic beverages. In epidemiological studies, it has been assumed that one cup of coffee contains at least $100 \mathrm{mg}$ of caffeine, and soft drinks, such as colas, contain 10-50 mg of caffeine per 12-ounce serving (Christian \& Brent, 2001).
Kenya is a major producer of coffee in Africa, and caffeine consumption is ranked second after tea in Kenya. Sources of caffeine consumed in Kenya range from packaged non-alcoholic beverages to over the counter caffeinated drugs. There is lack of information on the amount of caffeine consumed by an adult Kenyan in one serving of a mug of brewed coffee or a packaged caffeinated soda. Many consumers of soft and energy drinks are totally unaware of caffeine content in these drinks. Similarly, commercially available non-alcoholic beverages served in Kenya do not have product label on the packaging material and do not even declare caffeine as part of the ingredients.

In Kenya, the major source of caffeine is coffee. Kenyans consume only 3,000 tons of 50,000 tons of coffee grown in the country annually (Douglas, 2009). However, the trend is changing with increase in coffee consumption in urban centers.

The present study determined the caffeine contents of commonly consumed non-alcoholic drinks sold in supermarkets in Nairobi Kenya using reverse phase high performance liquid chromatography with UV/VIS detector.

\section{METHOD}

\section{Chemicals and Apparatus}

A caffeine standard $99 \%$ pure purchased from Prolabo, ECC, Manchester M, Discovery ${ }^{\circledR}$ HS C- 18 column $25 \mathrm{~cm}$ x $4.6 \mathrm{~mm}$ x $5 \mu \mathrm{m}$ Cat. No. 568523 from Supelco, Sigma-Aldrich Co. USA and analytical grade methanol of 99.9\% purity from Panreac Quimica SAU, Barcelona, and HPLC grade water Fischer Scientific were used.

The stocks solutions and the extracts were mixed in a sonicator (Bandelin, Sonorex RK 255 transistor Berlin-German) and UV-Vis spectrophotometer (UV-1700, Pharmaspec Shimadzu, Tokyo Japan) was used to determine the wavelength of maximum absorption for caffeine. HPLC fitted with UV/VIS detector (Shimadzu Corporation, Kyoto Japan) was used for the determination of amount of caffeine from the extracts. 


\section{Stock solutions}

A stock solution of $1.0 \mathrm{mg} / \mathrm{ml}$ of caffeine was prepared by weighing $100 \mathrm{mg}$ of caffeine into a $100 \mathrm{ml}$ volumetric flask. This was dissolved in distilled water to make up volume to $100 \mathrm{~mL}$. Working solution of $0.05 \mathrm{mg} /$ $\mathrm{mL}, 0.1 \mathrm{mg} / \mathrm{mL}, 0.5 \mathrm{mg} / \mathrm{mL}$ were made by pipetting 5,10 and $50 \mathrm{~mL}$ of stock solution into a $100 \mathrm{ml}$ volumetric flask and topping up the volume to $100 \mathrm{~mL}$ with HPLC analytical grade water. These serial dilutions of the standards were filtered using $0.45 \mu \mathrm{m}$ membrane filters and used in the construction of the calibration curve.

The mobile phase was prepared daily by mixing 80 volumes of methanol and 20 of HPLC water. This was then filtered under vacuum through $0.45 \mu \mathrm{m}$ pore size nylon filters and put in the HPLC mobile phase reservoir.

\section{Sample Preparation}

Nineteen samples of non-alcoholic energy drinks and prepared teas were degassed in a sonicator for 10 minutes and $5 \mathrm{~mL}$ of each pipetted into a $100 \mathrm{~mL}$ amber flask and diluted 20 times with mobile phase (methanol: water at $80: 20 \mathrm{v} / \mathrm{v}$ ). An aliquot of $2-5 \mathrm{~mL}$ diluted sample was filtered through membrane filter of $0.45 \mu \mathrm{m}$ pore diameter to remove any particulate matter. The filtrate was ready for determination of caffeine content by HPLC.

\section{UV/Visible Spectra Scan for caffeine}

Dilute caffeine standard stock solution was scanned at a range of 190 to $350 \mathrm{~nm}$ wavelength with UV-Vis spectrophotometer (UV-1700, Pharmaspec Shimadzu, Tokyo Japan) to generate complete absorbance spectra and identify wavelength of maximum absorbance for subsequent use in HPLC-UV/VIS detector.

\section{Determination of caffeine content by HPLC-UV method}

The HPLC column was conditioned for 20 minutes with the mobile phase and the programmed oven temperature of $40^{\circ} \mathrm{C}$ before any analysis was carried out. HPLC-UV/VIS method was created with the following parameters, oven temperature of $40^{\circ} \mathrm{C}$, mobile phase flow rate of $1.5 \mathrm{~mL} /$ minute, detector wavelength of $272 \mathrm{~nm}$ and injection volume of $20 \mu \mathrm{L}$.

A blank sample composed of the extraction solution was injected severally until the baseline was good. Then, serially diluted standards were injected in triplicates, and the retention time, peak areas and heights were recorded. After cleaning the system with blank solution, diluted sample was injected three times and the peak height and area with retention time corresponding to that of the standard was recorded. The chromatograms for caffeine standard and a sample of Coca cola beverage are shown in Figures 1 and 2 respectively.

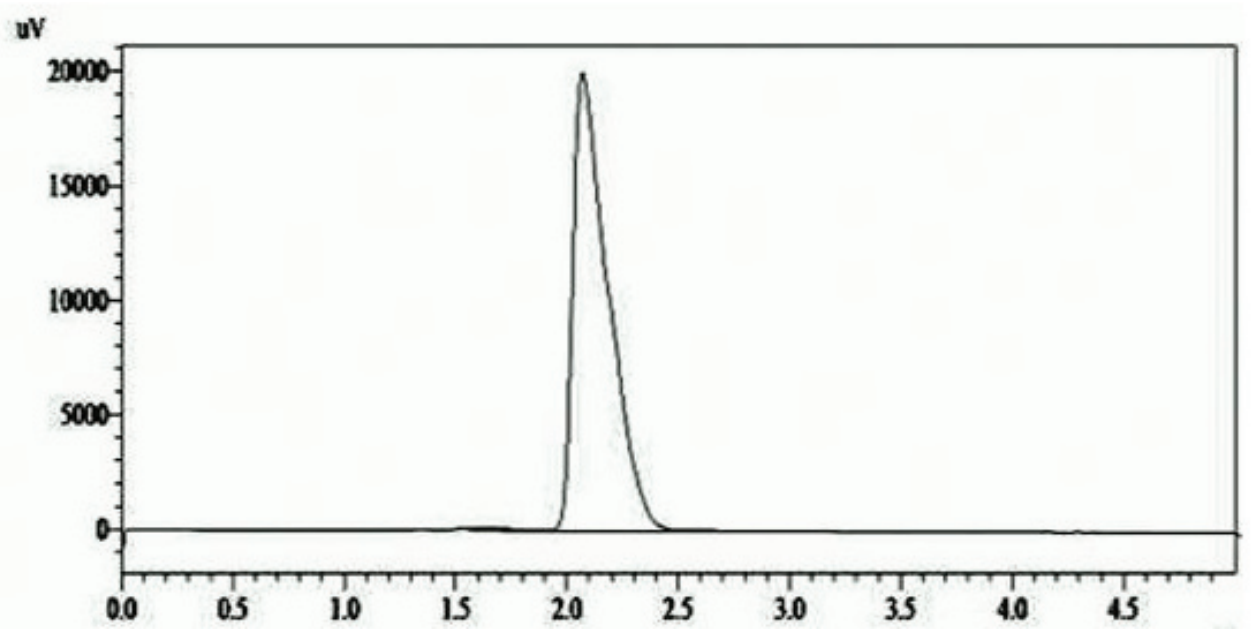

Figure 1. $10 \mathrm{ppm}$ caffeine standards chromatogram, with retention time at 2.1 minutes 


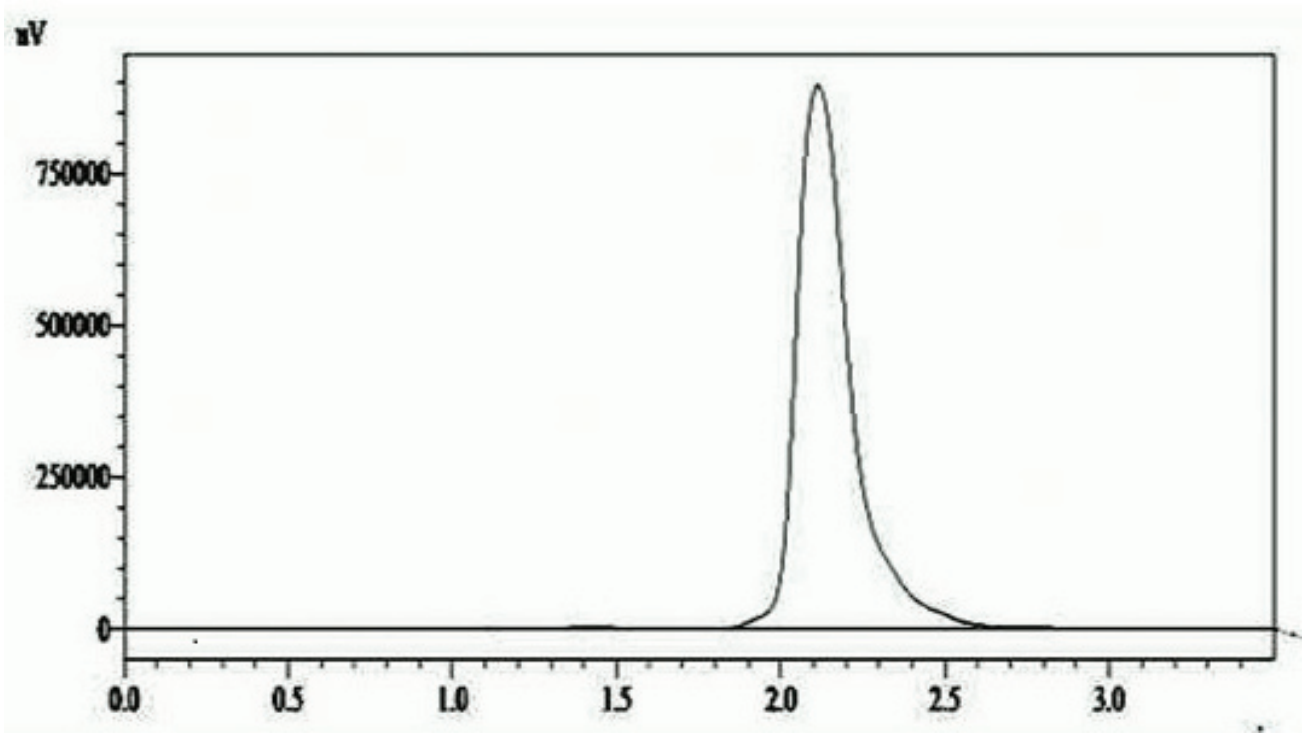

Figure 2. Corresponding peak for caffeine from Coca cola drink. The retention time is 2.1 minutes

\section{RESULTS}

The wavelength of maximum absorbance for caffeine was found to be $272 \mathrm{~nm}$. This was then programmed in the HPLC-UV detector for the analysis of caffeine content in nonalcoholic beverages and prepared teas. The mean retention time for caffeine was found to be 2.11 minutes with a standard deviation of \pm 0.03 minutes. After analysis of the serial dilutions of the caffeine standard the coefficient of correlation was found to be 0.9993 , and the equation of the linear regression was sufficiently fit for quantitative determination of caffeine in the samples. This is shown in Figure 3.

The limit of detection (LOD) and quantitation (LOQ) of the analytes were determined based on the standard deviation of the detector response $(\alpha)$ and the slope $(\mathrm{m})$ of the calibration curve. The following formulas were used; $3 \alpha / \mathrm{m}$ and $10 \alpha / \mathrm{m}$ for LOD and LOQ respectively (ISO 11843-2:2003). The limit of detection and quantitation of caffeine according to the method used were found to be 0.279 and 0.931 $\mu \mathrm{g} / \mathrm{mL}$, respectively as shown in Table 1 .

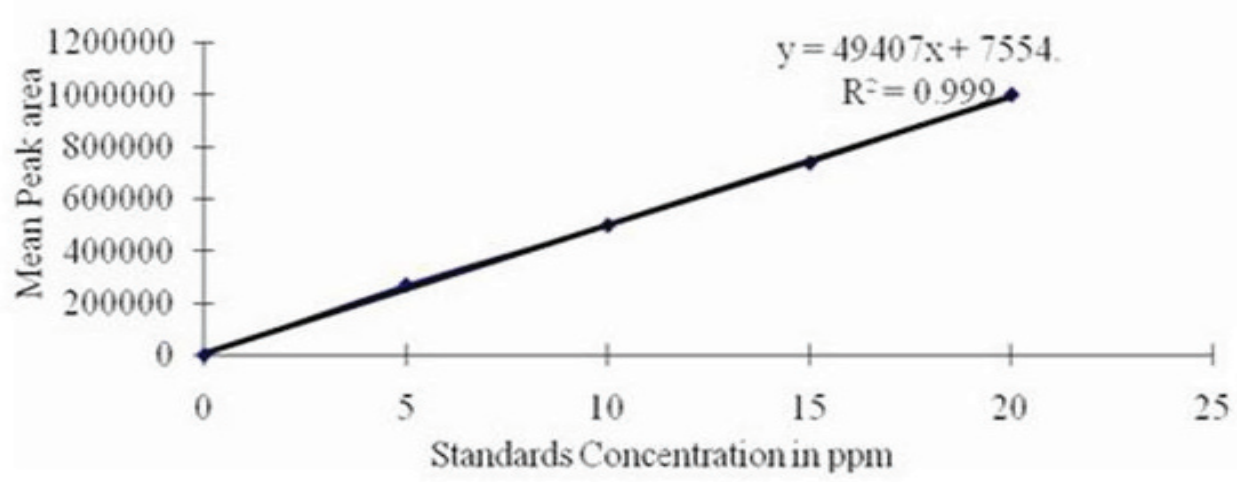

Figure 3. Linear regression curve for the serially diluted standards of caffeine 
Table 1. Summary of precision and validation data for caffeine in non-alcoholic beverages obtained with reverse phase $\operatorname{HPLC}(n=12)$

\begin{tabular}{ll}
\hline Validation Parameter & Amount in $(\boldsymbol{\mu g} / \mathbf{m L})$ \\
\hline Limit of detection (LOD) & 0.279 \\
Limit of quantitation (LOQ) & 0.931 \\
\hline
\end{tabular}

The limit of detection and quantitation of the method were determined by $3 \alpha / \mathrm{m}$ and $10 \alpha / \mathrm{m}$, where $\alpha$ stands for standard deviation of the detector response and $\mathrm{m}$ is the slope of the linear regression curve. The detector response was obtained from 6 injections of $10 \mathrm{ppm}$ caffeine standard and then this concentration was divided by the average peak area.

The ruggedness of the HPLC method was determined by analysis of a known concentration of the standard working solution in the same chromatographic system and column on three different days. This is shown in Table 2. The prepared mixture of standard was injected twelve times each day for a period of three days by the same analyst. The retention times and peak areas symmetry were found to be consistent during the three days analysis with the $0.009 \%$ and $0.302 \%$ relative standard deviations for the peak area and retention times respectively. The comparable detector responses obtained on different days indicate that the method is capable of producing consistent data for routine analysis.

Analysis of 19 samples found that XL energy drink had the highest amount of caffeine with $98.06 \mathrm{mg}$ in a $250 \mathrm{~mL}$ volume. This was closely followed by Burn which had $97.55 \mathrm{mg}$ per $250 \mathrm{ml}$. The results for the other samples analyzed are as shown in Table 3. Tango orange, 7UP and Rani Float were found not to contain caffeine.

\section{DISCUSSION}

Scientific literature is rich with a wide range of values for caffeine content in food products. In United States the following standard values are suggested by several authors, coffee $(5 \mathrm{oz})$ $85 \mathrm{mg}$ for ground roasted coffee, $60 \mathrm{mg}$ for instant and $3 \mathrm{mg}$ for decaffeinated, $18 \mathrm{mg}$ for instant colas. Caffeine consumption survey data are limited. In US approximately a mean daily caffeine intake for consumers is $4 \mathrm{mg} / \mathrm{kg}$ and among children younger than 18 years of age who are consumers of caffeine-containing foods, the mean daily caffeine intake is about $1 \mathrm{mg} / \mathrm{kg}$ (Barrone \& Roberts, 1996).

Caffeine use is associated with several distinct psychiatric syndromes such as caffeine intoxication, withdrawal, dependence, caffeine-induced sleep disorder, and caffeineinduced anxiety disorder. In certain people, caffeine consumption causes adverse health effects, such as anxiety, palpitations, irritability, difficulty sleeping and stomach upsets. Other studies have indicated moderate caffeine intake could lower consumers' risk of liver disease (Frary et al., 2005). The American Dietetic Association and the UK Food Standards Agency recommend not more than $300 \mathrm{mg}$ of caffeine per day (Frary et al., 2005). This recommendation is particularly useful to pregnant women who have greater

Table 2. Day to day peak area and \% recovery of $10 \mathrm{ppm}$ caffeine standard

\begin{tabular}{llll}
\hline Number of days & $\mathbf{1}$ & $\mathbf{2}$ & $\mathbf{3}$ \\
$*$ Mean Area \pm SD & $524112 \pm 3505$ & $529257 \pm 4966$ & $525715 \pm 3842$ \\
Percent RSD & 0.007 & 0.009 & 0.007 \\
\hline
\end{tabular}

$\mathrm{SD}=$ standard deviation; $\mathrm{RSD}=$ relative standard deviation.

* Mean of $10 \mathrm{ppm}$ spectral peak areas for caffeine standard injected daily was used to monitor the reproducibility of the method. 
Table 3. Summary of caffeine contents in $\mathrm{mg} /$ total can volume in $\mathrm{mL}$ of various beverages

\begin{tabular}{ll}
\hline Non-alcoholic beverage & $\begin{array}{l}\text { Caffeine conc. } \\
\text { In mg per can }\end{array}$ \\
\hline XL energy drink $250 \mathrm{ml}$ & 98.06 \\
Burn $250 \mathrm{ml}$ & 97.55 \\
Red Bull $250 \mathrm{ml}$ & 93.54 \\
Shark $250 \mathrm{ml}$ & 91.84 \\
Bomba $250 \mathrm{ml}$ & 91.20 \\
Atomic $250 \mathrm{ml}$ & 90.52 \\
B-52 250ml & 79.45 \\
Safari ice tea $310 \mathrm{ml}$ & 66.29 \\
Pepsi Maxi $500 \mathrm{ml}$ & 46.26 \\
Bullet $250 \mathrm{ml}$ & 44.71 \\
Africola $500 \mathrm{ml}$ & 42.25 \\
M-150 150ml & 38.50 \\
Vigor Power ice $250 \mathrm{ml}$ & 36.84 \\
Coca cola $330 \mathrm{ml}$ & 33.27 \\
Strong Horse $250 \mathrm{ml}$ & 6.19 \\
Tango cherry $330 \mathrm{ml}$ & 0.23 \\
Tango orange $330 \mathrm{ml}$ & 0 \\
7 UP 500ml & 0 \\
Rani float & 0 \\
\hline & \\
\hline
\end{tabular}

risk of miscarriage or giving birth to low birth weight babies.

Because of the health concerns associated with caffeine consumption, it is important that the manufacturers of caffeinated non-alcoholic beverages and teas append appropriate warning labels declaring presence and amount of caffeine. Studies show that $30 \mathrm{mgs}$ or less of caffeine can alter self-reports of moods and affect behavior and $100 \mathrm{mg}$ per day can lead to physical dependence and withdrawal symptoms upon abstinence.

Caffeine is an addictive drug, which alters the brain's natural state and stimulates it in a manner similar to the amphetamines, cocaine and heroin. One challenge with caffeine addiction is that most people don't think of caffeine as a drug.

Determination of caffeine content in nonalcoholic beverages and prepared teas is a very important analytical process to safeguard the well-being of the people who are vulnerable to the adverse effects of caffeine.

\section{CONCLUSIONS}

Reverse phase high performance liquid chromatography method for determination of caffeine content in non-alcoholic beverages is simple, accurate and robust. The amount of caffeine in non-alcoholic beverages is critical especially when one what to control consumption of caffeine. The data obtained in this study is important in the local setting because most of these non-alcoholic beverages list caffeine in their product label without withholding the amount contained. Therefore, one can easily control caffeine intake if caffeine is declared as an ingredient and at the same time the amount is indicated in the packaging materials.

\section{ACKNOWLEDGEMENTS}

The authors wish to thank Kenya Bureau of Standards for availing the equipment in Chromatography Laboratory and reagents for this study. The information from this study should be taken as the views of the authors and not of the Kenya Bureau of Standards.

\section{REFERENCES}

Barone J. J, \& Roberts H. R. (1996). Caffeine consumption. Food and Chemical Toxicology, 34, 119-129.

Bolton, S., \& Garry, N. (1981). Caffeine-Psychological Effects, Use and Abuse. Journal of Orthomolecular Psychiatry, 210 (3), 202-21.

Chou, K. H., \& Bell, L. N. (2007). Caffeine content of prepackaged national-brand 
and private-label carbonated beverages. Journal of Food Science, 72 (6), C33742.

Christian, M. S., \& Brent, R. L. (2001). Teratogen update: Evaluation of the reproductive and developmental risks of caffeine. Journal of Teratology, 64, 51-78.

Dawber, T.R., Kannel, W. B., \& Gordon, T. (1977). Coffee and cardiovascular disease. Observations from the Framingham study New England Journal of Medicine, 291, 871-874.

Douglas Hyde. (2009). Paying for the emergence by displacing the settlers: Global coffee and rural restructuring in late colonial Kenya. Journal of Global History, 4, 81-103

Frary, C. D., Johnson R. K., \& Wang M. Q. (2005). Food sources and intakes of caffeine in the diets of persons in the United States. Journal of the American Dietetic Association, 105,110-113.

Klatsky, A. L., Armstrong, M. A., \& Friedman, G. D. (1993). Coffee, tea and mortality. Annals of Epidemiology, 3, 375-381.

Knight, C A., Knight I., Mitchell D. C., \& Zepp J. E. (2004). Beverage caffeine intake in US consumers and subpopulations of interest: estimates from the Share of Intake Panel survey. Food Chemistry and Toxicology, 42: 1923-1930.

Nehlig, A., Daval, J. L., \& Debry, G. (1992). Caffeine and the central nervous system: mechanisms of action, biochemical, metabolic and psychostimulant effects. Brain Research Review, 17 (2): 139-70.

Schreiber, G. B., Robins, M., Maffeo, C. E., Masters, M. N., Bond, A. P.,

Morganstein, D. (1988). Confounders contributing to the reported associations of coffee or caffeine with disease. Preventive Medicine, 17, 295-309.

Watson, J., \& Kerr, D. (1999). The best defense against hypoglygemia is to recognize it: is caffeine useful? Diabetes Technology and Therapeutics, 1, 193-200.

Winkelmayer, W. C., Stampfer, M. J., Willet, W. C., \& Curhan, G. C. (2005).

Habitual caffeine intake and the risk of hypertension in women. Journal of the American Medical Association, 294, 2330-2335.

Yano, K., Rhoads, G.G., \& Kagan. (1977). Coffee, alcohol, and risk of coronary heart disease among Japanese men living in Hawaii. New of England Journal Medicine. 297, 405-409. 
